PAEDIATRIC LUNG DISEASE

\title{
The role of neutrophil apoptosis in the resolution of acute lung injury in newborn infants
}

\author{
S Kotecha, R J Mildner, L R Prince, J R Vyas, A E Currie, R A Lawson, M K B Whyte
}

Thorax 2003;58:961-967

See end of article for authors' affiliations .....................

Correspondence to: Dr Sailesh Kotecha, Department of Child Health, University of Leicester, Leicester LE2 7LX, UK; sk43@le.ac.uk

Received 25 October 2002 Accepted for publication 29 July 2003
Background: The persistent airway neutrophilia observed in chronic lung disease of prematurity (CLD) may reflect inappropriate suppression of neutrophil apoptosis.

Methods: 134 bronchoalveolar lavage (BAL) samples were obtained from 32 infants requiring mechanical ventilation for respiratory distress syndrome (RDS): 13 infants (median gestation 26 weeks, range 23 to 28) subsequently developed CLD (CLD group), and 19 infants (gestation 31 weeks, range 25 to 39) recovered fully (RDS group). A further $73 \mathrm{BAL}$ samples were obtained from 20 infants (median age 2 days, range 1 to 402) receiving extracorporeal membrane oxygenation (ECMO) for severe respiratory failure. Results: Neutrophil apoptosis was increased in the RDS group (mean (SEM) neutrophil apoptosis on day 7 BAL: RDS $17.0(8.6) \% v$ CLD $0.7(0.2) \%(p<0.05))$. BAL fluid obtained from RDS but not CLD patients was proapoptotic to neutrophils (apoptosis ratio BAL fluid/saline control: day 1, RDS 9.8 (5.5) v CLD 1.2 (0.1) $(p<0.05)$; day 2, RDS 4.32 (2.8) v CLD $0.5(0.4)(p<0.05))$. There were similar findings in the ECMO group: survivors had proapoptotic BAL fluid compared with non-survivors (apoptosis ratio day 1 , survivors 7.9 (2.1) v non-survivors $2.1(0.7)(p<0.05))$.

Conclusions: Inappropriate suppression of neutrophil apoptosis may be associated with a poor outcome in newborn infants with respiratory failure.
$\mathrm{T}$ he neutrophil granulocyte is a major participant in the acute inflammatory response in tissues, being recruited from the circulation when local defences are overwhelmed. The constitutively short life span of the neutrophil is less than 24 hours in the circulation, and these cells are exquisitely sensitive to apoptosis (programmed cell death). ${ }^{12}$ Apoptosis is important in the normal resolution phase of inflammation, as it leads to functional downregulation ${ }^{3}$ and to recognition and clearance of the apoptotic neutrophils by macrophages. ${ }^{1}$ Neutrophil life span is extended in tissues ${ }^{4}$ and this, together with modulation of neutrophil apoptosis by cytokines and other inflammatory mediators, ${ }^{56}$ suggests active control of cell death during the course of inflammation in vivo. Apoptosis of neutrophils present within the lung is inappropriately suppressed in patients with acute respiratory distress syndrome (ARDS), and the bronchoalveolar lavage (BAL) fluid of such patients is antiapoptotic to human neutrophils. ${ }^{7}$ This antiapoptotic effect was observed to be maximal in the early stages of $\mathrm{ARDS}^{7}$ and to decline at later time points. The suppression of apoptosis is predominantly mediated through elaboration of antiapoptotic cytokines. ${ }^{7}$

We have previously found that neutrophils that have been "aged" in vitro but have not undergone apoptosis show equivalent proinflammatory functions to freshly isolated neutrophils, emphasising the potential of these cells to cause tissue damage. ${ }^{3}$ We have also reported that airway neutrophilia, shown on BAL studies, is associated with the development of chronic lung disease of prematurity (CLD) in preterm infants who require mechanical ventilation for neonatal respiratory distress syndrome (RDS). ${ }^{9}$ We hypothesised that the persistent airway neutrophilia observed in CLD is caused by inappropriate suppression of neutrophil apoptosis. We therefore investigated neutrophil apoptosis in infants who developed CLD following neonatal RDS (CLD group) compared with those who recovered from RDS (RDS group). Specifically, we investigated the proportion of apoptotic neutrophils in bronchoalveolar lavage fluid at time points up to 14 days in infants in the two groups receiving mechanical ventilation; and whether BAL fluid from the CLD and RDS groups differs in its effects on constitutive neutrophil apoptosis in vivo.

\section{METHODS}

\section{Patient groups}

Infants with and without respiratory failure were recruited into the study and divided into the following groups:

- Chronic lung disease of the newborn (CLD group): these infants initially required mechanical ventilation for RDS. At 28 days of age they remained oxygen dependent and had persistent chest $x$ ray abnormalities.

- Respiratory distress syndrome (RDS group): these infants were also ventilated for RDS but by 28 days were nursed in air and had a normal chest $x$ ray.

- Extracorporeal membrane oxygenation (ECMO group): these were infants with severe respiratory failure as a result of various conditions, including RDS, meconium aspiration syndrome, and persistent pulmonary hypertension of the newborn. Of those recruited, 14 survived and six did not.

- Controls: these infants received mechanical ventilation perioperatively for surgical conditions for non-pulmonary reasons, and were exposed to less than $28 \%$ inspired oxygen.

Infants eligible for the CLD, RDS, and control groups but with proven or strongly suspected sepsis were excluded from the study. The diagnosis of sepsis was based on one or more

\footnotetext{
Abbreviations: ARDS, acute respiratory distress syndrome; BAL, bronchoalveolar lavage; CLD, chronic lung disease of prematurity; ECMO, extracorporeal membrane oxygenation; GM-CSF, granulocyłemacrophage colony stimulating factor; IL, interleukin; RDS, respiratory distress syndrome; TNF $\alpha$, tumour necrosis factor $\alpha$; TRAIL, tumour necrosis factor related apoptosis inducing ligand; TUNEL, TdT mediated dUTP-biotin 3'-OH nick-end labelling
} 
of the following: maternal fever or infection, prolonged rupture of membranes ( $>48$ hours), positive blood cultures in the infant, positive endotracheal cultures, and raised or relative increase in blood white cell count or $\mathrm{C}$ reactive protein in the face of clinical suspicion of sepsis. Six infants were excluded from the study because organisms were identified from secretions obtained through the endotracheal tube or because of central line sepsis.

Fully informed, written consent was obtained from the parents of each infant. The study was approved by the Leicestershire Health Authority research ethics committee.

\section{Bronchoalveolar lavage}

BAL was done twice weekly in the ventilated preterm infants until 21 days of age or until extubation. In the infants receiving ECMO, BAL was done daily for the first four days of treatment. BAL was undertaken as previously described. ${ }^{9-11}$ Briefly, with the infant lying supine and the head turned to the left, a $5 \mathrm{~F}$ catheter was gently inserted into the endotracheal tube until resistance was felt. Two aliquots of $1 \mathrm{ml}$ per $\mathrm{kg}$ of saline $(0.9 \%)$ were instilled through the catheter and immediately sucked back. The BAL samples were processed within 20 minutes of the lavage procedure. From the pooled aspirate, aliquots were taken for total and differential cell count. The remainder of the aspirate was centrifuged at $500 \times g$ for 10 minutes at room temperature and the supernatant stored at $-70^{\circ} \mathrm{C}$ for later analysis. All subsequent analyses were done by investigators who were blinded to the clinical diagnosis in each case.

\section{Characterisation of BAL cells}

Haemocytometer counting was used to obtain total cell counts per ml of BAL fluid. Cytocentrifuge preparations were made from a further aliquot of BAL fluid. At least 300 cells were counted on each cytospin to provide a differential cell count. In addition, apoptotic neutrophils, assessed morphologically by the typically condensed nuclei, were counted and expressed as a percentage of the total neutrophil count. As all the counts were done before classification of the infants to the retrospective diagnoses of CLD or RDS, the counts were blind to the outcome.

\section{Isolation of neutrophils}

Human peripheral blood neutrophils were obtained from healthy adult volunteers. Ethical approval was obtained from the South Sheffield research ethics committee and all subjects gave fully informed consent. Neutrophils were isolated from citrated venous blood by dextran sedimentation and centrifugation through a discontinuous plasma-Percoll gradient (Amersham Pharmacia Biotech, Buckinghamshire, UK) as previously described. ${ }^{12}$ Purity was assessed by counting $>500$ cells on duplicate cytospin preparations and was always $>95 \%$, with contaminating cells being almost exclusively eosinophils.

\section{Assessment of neutrophil apoptosis in BAL}

The apoptotic activity of the BAL fluid was assessed as previously described. ${ }^{7}$ Neutrophils were suspended at a concentration of $3.5 \times 10^{6} / \mathrm{ml}$ in Roswell Park Memorial Institute medium (RPMI), with $10 \%$ fetal calf serum and with penicillin and streptomycin (100 U/l) (all from Life Technologies Inc, Glasgow, UK).$^{13}$ The "age" of neutrophils in culture was calculated, designating this stage as time 0 . A $100 \mu \mathrm{l}$ aliquot of the neutrophil suspension was added to $100 \mu \mathrm{l}$ of BAL fluid from the newborn infants, with normal saline used as control. Cells were incubated in 96-well Falcon "Flexiwell" plates (not tissue culture treated; BD Pharmingen, Cowley, Oxford, UK) at $37^{\circ} \mathrm{C}$ in a $5 \% \mathrm{CO}_{2}$ atmosphere for five hours. A five hour time point was chosen because there is little (typically less than 5\%) constitutive apoptosis in control (untreated) populations, so a proapoptotic effect of BAL fluid upon neutrophils should be

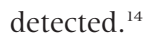

At five hours, apoptosis was quantified by assessment of nuclear morphology on Giemsa-stained cytocentrifuge preparations, counting $>300$ cells per slide on duplicate cytospins. ${ }^{13}$ This method has been shown to correlate closely with other measurements of neutrophil apoptosis, including Annexin $\mathrm{V}$ binding ${ }^{15}$ and shedding of CD16. ${ }^{16}$ In addition, necrosis was assessed at all time points by exclusion of the vital dye trypan blue, and was less than $2 \%$ unless otherwise indicated. In the results section, the apoptotic activity of BAL fluid is expressed as a ratio of the value in the saline control in the same experiment.

\section{Cytokine assays}

The concentrations of tumour necrosis factor $\alpha(\mathrm{TNF} \alpha)$, granulocyte-macrophage colony stimulating factor (GMCSF), and interleukin (IL)-10 were measured in BAL fluid using commercially available enzyme linked immunosorbent assays (ELISA) according to the manufacturer's instructions (R\&D Systems, Oxfordshire, UK).

\section{Statistical methods}

Gestation and birth weight are expressed as the median values and range. Only one sample was obtained from any individual infant at any one time point, and the mean of results from two cytospins is given. Total and differential cell counts and the apoptotic activity of BAL fluid are expressed as mean (SEM) of the number ( $\mathrm{n}$ ) of independent experiments, with each experiment using cells from separate donors and carried out in duplicate. Data from the controls are only given for BAL fluid neutrophil apoptotic activity (see fig $4 \mathrm{~A}$ ) as too few cells were present on the cytocentrifuge preparations from these infants for reliable differential cell counts to be obtained.

The apoptotic activity of BAL fluid is given relative to that obtained for the saline control. Thus values of $>1.0$ imply proapoptotic activity and $<1.0$ imply antiapoptotic activity in BAL fluid relative to saline.

The Mann-Whitney U test was used for comparisons between patient groups, as the data were non-parametrically distributed. Cell count data were compared by analysis of variance (ANOVA) with the Bonferroni post-test, using the Prism 3.0 program (GraphPad Software Inc, San Diego, California, USA). Maximum values for each infant for BAL fluid neutrophil activity during the first week of age were also compared between the RDS and CLD groups. Correlations between gestation, birth weight, and cytokines with BAL neutrophil apoptotic activity were determined using the Pearson correlation coefficient ( $R$ value). Results are considered statistically significant at a probability $(\mathrm{p})$ value of $<0.05$.

\section{RESULTS}

\section{Patient characteristics}

We obtained 134 BAL samples from 32 infants in the neonatal unit. The characteristics of these infants are shown in table 1. BAL samples were obtained from the CLD group at time points up to 21 days. For the RDS group, data are shown only up to 14 days for some of the analyses, as thereafter there were too few samples available. No clinical differences were noted between infants who were or were not treated with antenatal corticosteroids. There were no significant differences between the two groups for inspired oxygen or ventilation variables during the first day of life. However, when the gestational age and birth weight were compared there were differences between the two groups (birth weight, 


\section{Table 1 Patient characteristics}

\begin{tabular}{lll}
\hline & CLD & RDS \\
\hline Number & 13 & 19 \\
Gestation (weeks) & $26(23$ to 28$)$ & $31(25$ to 39$) \dagger$ \\
Birth weight (g) & $750(630$ to 1070$)$ & $1400(720$ to 4160$) \dagger$ \\
Antenatal dexamethasone & $12 / 13(92 \%)$ & $10 / 19(53 \%)$ \\
Surfactant* & $12 / 13(92 \%)$ & $16 / 19(84 \%)$ \\
PIP (mean first 24 hours) & $17(14$ to 36$)$ & $16(11$ to 22$)$ \\
MAP (mean first 24 hours) & $8(4$ to 18) & $7(4$ to 13$)$ \\
Fio $_{2}$ (mean first 24 hours) & $0.37(0.24$ to 1.00$)$ & $0.32(0.21$ to 0.76$)$
\end{tabular}

${ }^{*}$ Curosurf ${ }^{\circledR}$, Chiesi Pharmaceuticals, Parma, Italy.

†Significant differences were noted between the two groups for birth weight and gestation (both $\mathrm{p}<0.001$ ).

$\mathrm{CLD}$, chronic lung disease; $\mathrm{Fio}_{2}$, inspired fractional oxygen; MAP, mean airway pressure; PIP, peak inspiratory pressure; RDS, respiratory distress syndrome.

$\mathrm{p}<0.001$; gestation, $\mathrm{p}<0.001$ ). We also studied nine control infants (median birth weight $2240 \mathrm{~g}$, range 900 to 3510; median gestation 36 weeks, range 26-40). These infants received mechanical ventilation but less than $28 \%$ inspired oxygen.

We obtained a further 73 BAL samples from 20 infants receiving ECMO (median age 2 days, range 1 to 402; median weight $3.7 \mathrm{~kg}$, range 2.5 to 10.0 ). Of these, 14 survived and six did not. BAL samples were obtained daily for the first four days of ECMO treatment. Of the survivors, six received ECMO for meconium aspiration syndrome, three for sepsis, two for congenital diaphragmatic hernia, and one each for pneumonia, persistent pulmonary hypertension, and respiratory distress syndrome. One infant with congenital diaphragmatic hernia, two with persistent pulmonary hypertension, and three with pneumonia did not survive.

\section{Total cell, absolute neutrophil, and alveolar macrophage cell counts}

The mean (SEM) total cell counts in BAL fluid from the RDS group increased from $258(74) \times 10^{4} / \mathrm{ml}$ on day $\mathrm{l}$ of age to 368 $(107) \times 10^{4} / \mathrm{ml}$ by day 4 , before decreasing rapidly to $<60.0 \times 10^{4} / \mathrm{ml}$ on days 10 and 14 (fig 1). In the CLD infants, however, the BAL fluid total cell counts were $124 \times 10^{4} / \mathrm{ml}$ at day 1 , increased gradually to $382(180) \times 10^{4} / \mathrm{ml}$ by day 10 , before declining to $193(105) \times 10^{4} / \mathrm{ml}$ by 17 days. There was a significant difference in BAL cellularity between the two

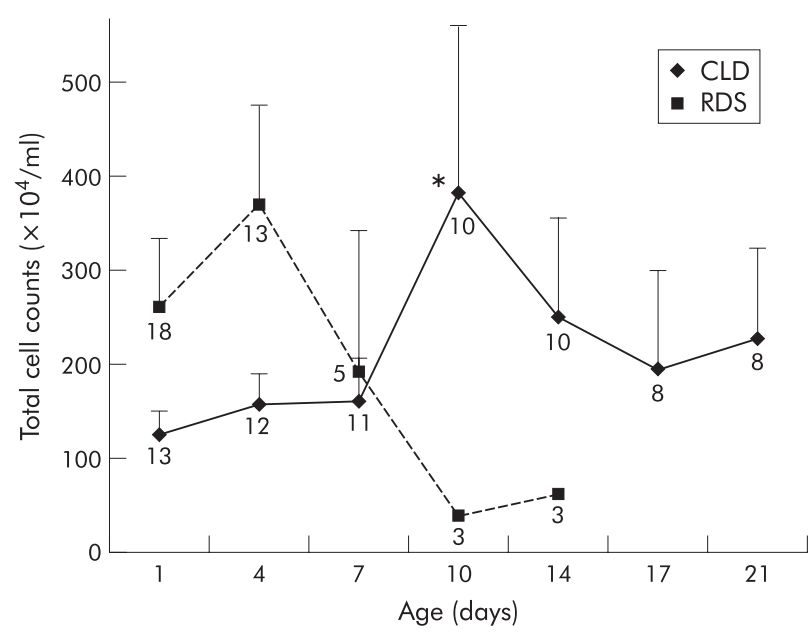

Figure 1 Total cell counts in bronchoalveolar lavage (BAL) fluid obtained from ventilated preterm infants who progressed to develop chronic lung disease of prematurity (CLD) and who recovered from the neonatal respiratory distress syndrome (RDS). The numbers beside each point are the number of BAL samples available for analysis. ${ }^{*} \mathrm{p}<0.05$.
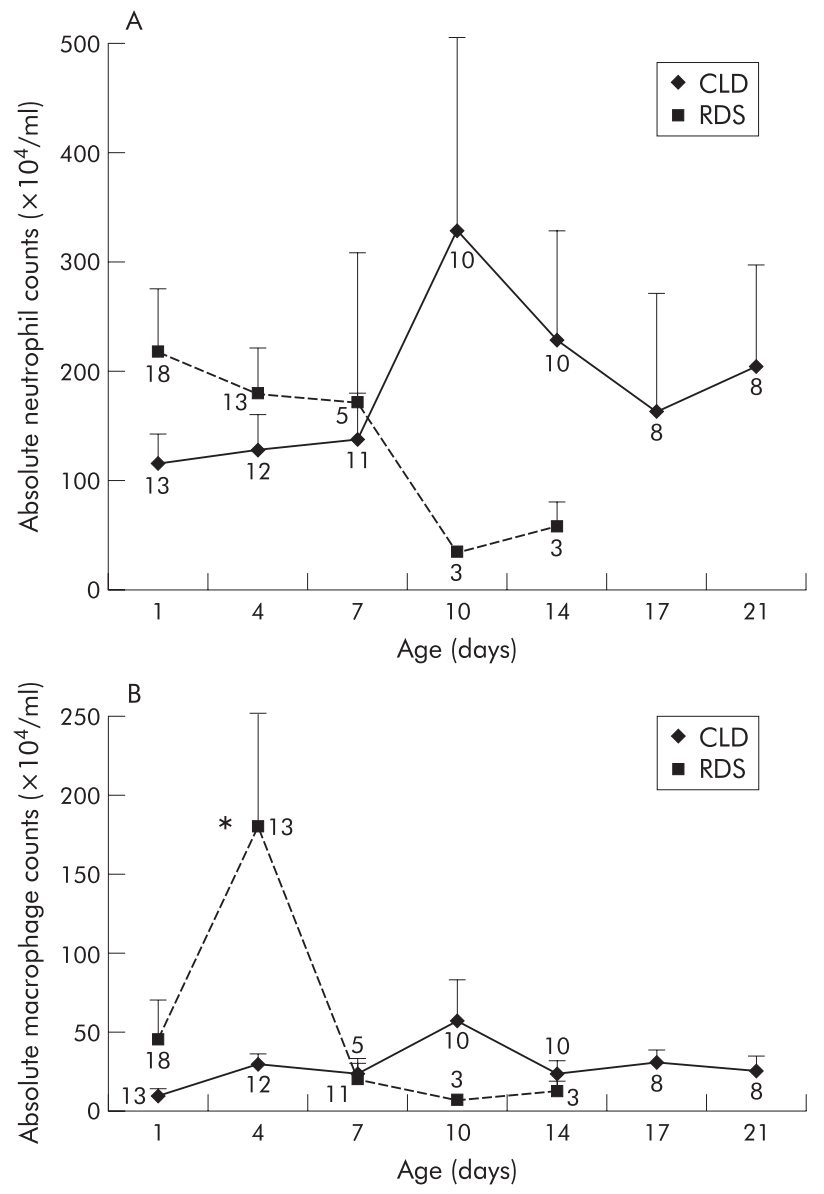

Figure 2 (A) Absolute neutrophil count and (B) alveolar macrophage count in bronchoalveolar lavage (BAL) fluid obtained from ventilated preterm infants who progressed to develop chronic lung disease of prematurity (CLD) and those who recovered from the neonatal respiratory distress syndrome (RDS). The numbers beside each point are the number of BAL samples available for analysis. ${ }^{*} \mathrm{p}<0.05$.

groups on day 10: RDS, $39(3.0) \times 10^{4} / \mathrm{ml} \quad v \quad \mathrm{CLD}, 382$ $(180) \times 10^{4} / \mathrm{ml}(\mathrm{p}<0.05)$.

Absolute BAL neutrophil counts in the RDS group decreased from $215(57) \times 10^{4} / \mathrm{ml}$ on day 1 to 176 $(40) \times 10^{4} / \mathrm{ml}$ on day 4 before decreasing to $<60.0 \times 10^{4} / \mathrm{ml}$ on days 10 and 14 (fig 2A). In the CLD infants, the neutrophil counts increased from $116(24) \times 10^{4} / \mathrm{ml}$ on day 1 to $326(180) \times 10^{4} / \mathrm{ml}$ on day 10 , before declining to 162 $(106) \times 10^{4} / \mathrm{ml}$ by day 17 . The difference in cellularity between the two groups was thus largely attributable to an increase in neutrophil numbers in the CLD group at day 10 (fig 2A), as we have shown in previous studies. ${ }^{9}$

BAL alveolar macrophage counts, in contrast, increased in the RDS group from $43(27) \times 10^{4} / \mathrm{ml}$ on day 1 to 177 $(74) \times 10^{4} / \mathrm{ml}$ on day 4 , before decreasing to $<15.0 \times 10^{4} / \mathrm{ml}$ thereafter between 7 and 14 days of age (fig 2B). In the CLD group, the alveolar macrophage count was $<30.0 \times 10^{4} / \mathrm{ml}$ on all days studied, except for a slight increase on day 10, to 56 $(26) \times 10^{4} / \mathrm{ml}$. Counts were significantly higher in the RDS group than the CLD group at day 4 (RDS, $177(74) \times 10^{4} / \mathrm{ml}$; CLD, $\left.29.0(6.5) \times 10^{4} / \mathrm{ml} ; \mathrm{p}<0.05\right)$ but thereafter no differences were found between the two groups.

\section{Assessment of neutrophil apoptosis in cells obtained by bronchoalveolar lavage}

The percentage of neutrophils that showed light microscopic features of apoptosis (fig 3A) was determined by a blinded 

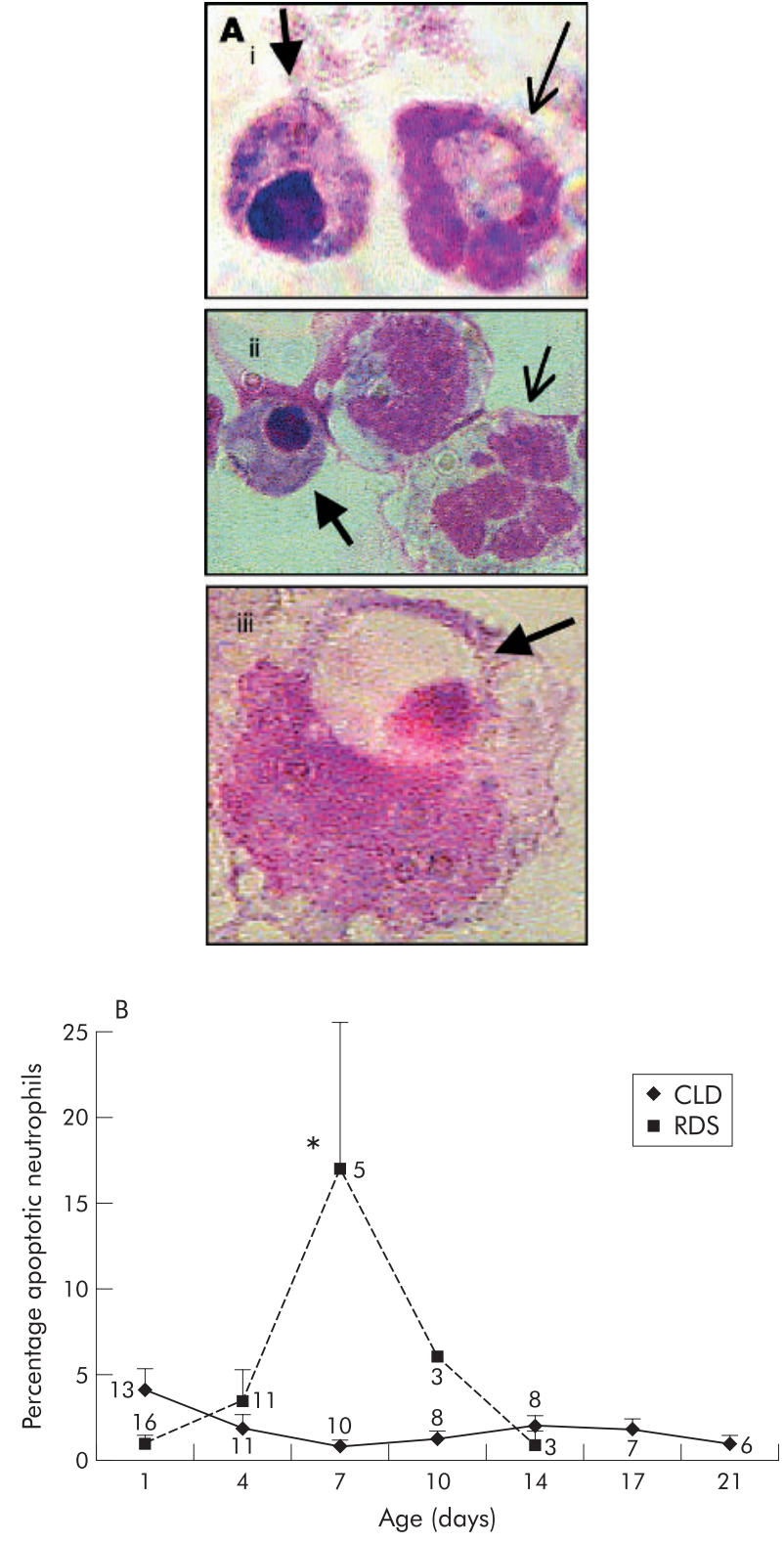

Figure 3 (A) Photomicrographs of cytocentrifuge slides prepared from bronchoalveolar lavage (BAL) fluid showing presence of apoptotic neutrophils (i, ii) and an alveolar macrophage engulfing an apoptotic neutrophil (iii). Solid arrowheads illustrate apoptotic neutrophils; these show morphological changes associated with apoptosis, including cell shrinkage and nuclear condensation. Unfilled arrowheads highlight healthy, non-apoptotic cells, which retain hypersegmented nuclear structure. (B) Percentage of apoptotic neutrophils shown for cells obtained from bronchoalveolar lavage in ventilated preterm infants who developed and recovered fully from the neonatal respiratory distress syndrome (RDS) $v$ those who progressed to develop chronic lung disease of prematurity (CLD). The numbers beside each point are the number of BAL samples available for analysis. ${ }^{*} \mathrm{p}<0.05$.

observer counting duplicate cytospins. The results are shown in fig 3B. In the RDS infants, the percentage of apoptotic PMN increased from $1.0(0.4) \%$ on day 1 to $17.0(8.6) \%$ on day 7 , before decreasing to $0.8(0.8) \%$ on day 14 of age. In contrast, the percentage of apoptotic neutrophils in BAL from infants developing CLD was 4.1 (1.2)\% on day 1 but $<1.8 \%$ thereafter until 21 days of age. At day 7 there was a significantly greater proportion of apoptotic PMN in the RDS group than in the CLD group $(\mathrm{p}<0.05)$.

\section{Modulation of neutrophil apoptosis by BAL fluid}

Incubation of peripheral blood neutrophils with day I BAL fluid from RDS infants resulted in an 11.0-fold induction in apoptosis relative to saline control. The proapoptotic effect of BAL fluid lessened gradually to $0.6(0.25)$ by day 7 (fig $4 \mathrm{~A}$ ). In contrast, the proapoptotic activity of BAL fluid from CLD infants was $<2.0$ throughout the 21 days studied. For
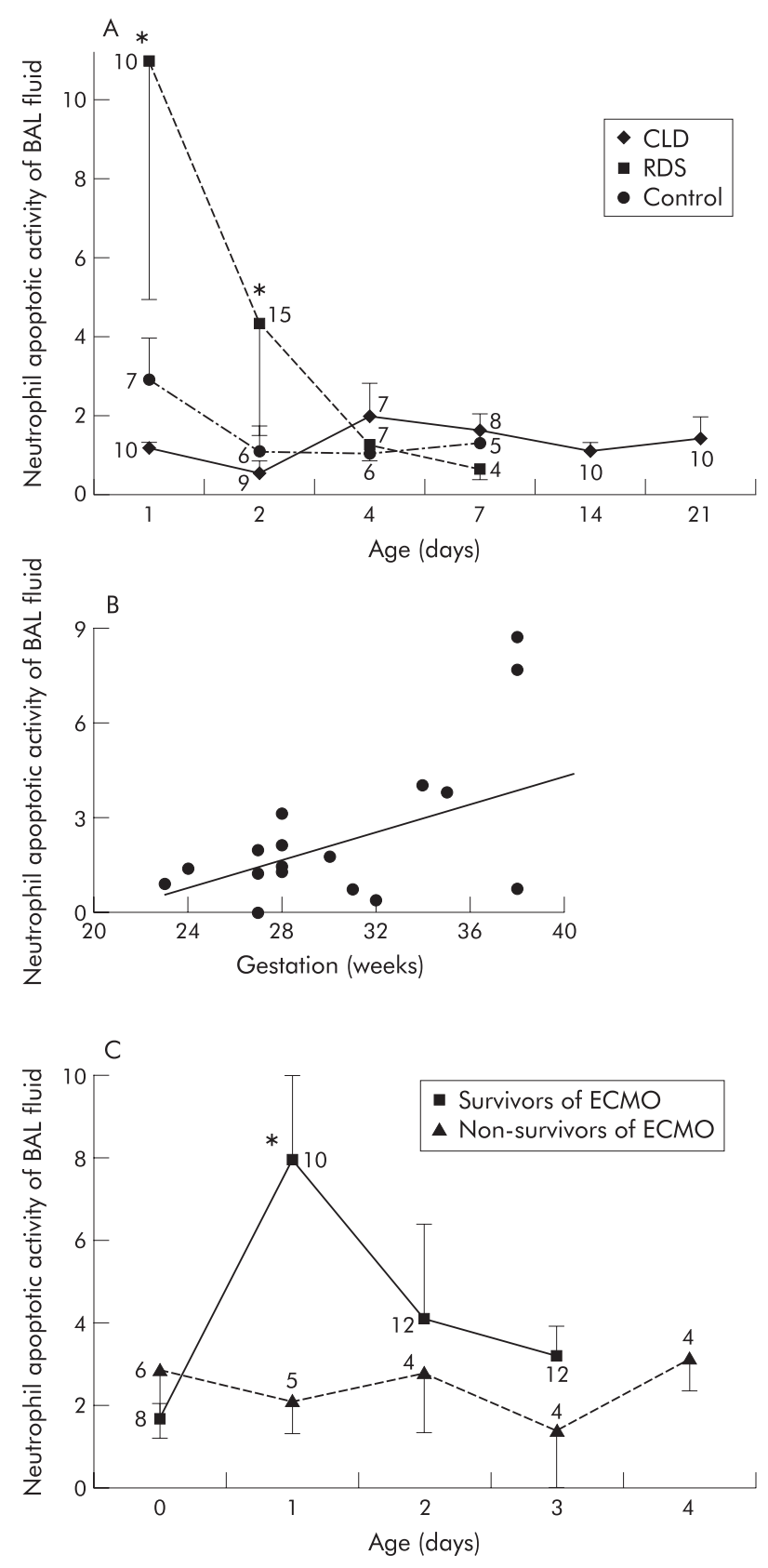

Figure 4 (A) Neutrophil apoptotic activity of bronchoalveolar lavage (BAL) fluid obtained from ventilated preterm infants who developed and recovered fully from the neonatal respiratory distress syndrome (RDS) and those who progressed to develop chronic lung disease of prematurity (CLD). (B) Moderate correlation between BAL fluid neutrophil apoptotic activity and gestational age $(R=0.50, \mathrm{p}<0.05)$. (C) Apoptotic activity of BAL fluid from infants who received extracorporeal membrane oxygenation for severe respiratory failure. The apoptotic activity of BAL fluid is expressed as a ratio of value for the saline control in the same experiment. The mean (SEM) percentage apoptosis for the saline control was $1.4(0.2) \%, n=10$. The numbers beside each point are the number of BAL samples available for analysis. ECMO, extracorporeal membrane oxygenation. ${ }^{*} \mathrm{p}<0.05$. 
comparison, BAL fluid obtained from control infants showed a mean 2.9-fold induction of apoptosis on day 1 , which fell to $<1.3$-fold thereafter until 7 days of age. The proapoptotic effect of RDS fluid on neutrophils was greater than that of CLD or control fluid on both day 1 and day $2(p<0.05)$.

We also analysed the data by comparing the maximum BAL fluid neutrophil activity during the first week of life in CLD and RDS infants, and noted a significant difference between the two groups: CLD, 1.3 (interquartile range (IQR), 0.8 to 2.0$) v$ RDS 1.9 ( 1.5 to 3.3$)(\mathrm{p}<0.05)$, mean difference $0.78,95 \%$ confidence interval 0.11 to 1.83 . The maximum value was achieved in the RDS infants at a median age of 2.0 days (IQR 1 to 2 ) and in the CLD infants at a later age of 4.0 days (IQR 2 to 7 ) (median difference 2.0, 95\% CI 0.001 to $3.000, \mathrm{p}<0.05)$. To determine if the BAL proapoptotic activity was related to either the gestation age or the birth weight of the infant, we compared gestational age and birth weight with the proapoptotic activity of the BAL fluid at day l. A correlation was found between the BAL fluid neutrophil activity at day $\mathrm{l}$ and gestational age (fig $4 \mathrm{~B})(R=0.5$, $\mathrm{p}<0.05)$ but not birth weight $(R<0.10, \mathrm{p}>0.05)$.

To further address the possibility that proapoptotic BAL fluid was associated with a better clinical outcome, we studied a cohort of infants who were treated with ECMO. We had previously shown a significant improvement in BAL neutrophilia in this cohort who survived the course of ECMO when compared with infants who did not. ${ }^{17}$ We hypothesised that those ECMO infants who survived might-as with the RDS cohort-have BAL fluid with a proapoptotic effect upon neutrophils and that this might be associated with resolution of acute lung injury. The BAL proapoptotic activity of ECMO survivors was $1.7(0.4)$ when placed on ECMO (day 0), increased to 7.9 (2.1) on day 1 , and then decreased to 3.1 (0.8) on day 3 of ECMO (fig 4C). In the infants who received ECMO but did not survive, the BAL fluid apoptotic activity was $<3.1$ throughout the treatment. On day 1 of ECMO treatment there was greater proapoptotic activity in BAL fluid of survivors than of non-survivors $(\mathrm{p}<0.05)$.

\section{Relation of soluble factors to apoptotic activity of BAL fluid}

Soluble factors present in BAL fluid can affect the survival of neutrophils. ${ }^{7}$ Because of the very small sample volumes obtained from the infants (typically less than $0.5 \mathrm{ml} / \mathrm{kg}$ ), we were limited to the study of a small number of candidate cytokines. We measured GM-CSF, as this has been shown to prolong neutrophil survival both in vitro ${ }^{5}$ and in vivo ${ }^{7}$; $\mathrm{TNF} \alpha$, which has a proapoptotic effect upon neutrophils at the time point studied ${ }^{18}$; and IL-10, because high levels of this anti-inflammatory cytokine are proapoptotic ${ }^{19} 20$ and are associated with a poorer outcome in patients with pneumonia. ${ }^{21}$ Both GM-CSF ${ }^{22}$ and $\mathrm{TNF} \alpha^{23}$ have been shown to be increased in infants who develop CLD compared with those who recover from RDS, and IL-10 may be deficient in infants who develop CLD. ${ }^{24}$

We measured BAL fluid levels of each of these cytokines by ELISA and correlated them with BAL fluid apoptotic activity in all the groups studied. All three cytokines were detected, either in all samples (TNF $\alpha$ ) or in the majority of samples. No relations were identified, however, between BAL levels of TNF $\alpha$, GM-CSF, or IL- 10 on day 1 and the numbers of apoptotic neutrophils present in BAL fluid at day 1 , nor with BAL neutrophil apoptotic activity at day 1 (all $R^{2}<0.25$, $\mathrm{p}>0.10$; data not shown). Comparisons were only made at day 1 as too few samples were available thereafter.

\section{DISCUSSION}

In these studies we showed that the development of chronic lung disease following neonatal respiratory distress syndrome is associated with persistent neutrophilia in BAL fluid, compared with preterm infants in whom RDS resolves, and we confirmed previous studies showing that this difference becomes apparent at day 10 of age. ${ }^{25}$ We hypothesised that the observed difference in neutrophil numbers could reflect differences in the regulation of neutrophil apoptosis between the two groups. We showed that the CLD group had reduced neutrophil apoptosis at day 7 compared with the RDS group, suggesting that increased survival may be a mechanism of neutrophil persistence in CLD. Furthermore, BAL fluid obtained from the RDS group was found to have a significant proapoptotic effect on peripheral blood neutrophils on days 1 and 2 of ventilation-an effect that was not seen in the CLD group. These results suggest that both the resolution of inflammation and a favourable clinical outcome were associated with higher rates of apoptosis and a proapoptotic lavage fluid.

We confirmed these findings in a second patient groupthose receiving treatment with ECMO-among whom we have previously shown a lack of resolution of pulmonary neutrophilia in the poor prognosis group (non-survivors). ${ }^{17}$ Again, a proapoptotic BAL fluid was associated with improved clinical outcome (that is, survival). Although the ECMO group was a heterogeneous group of patients in terms of their underlying diagnosis, with some having an inflammatory pathology (for example, pneumonia, meconium aspiration, RDS) and others not (persistent pulmonary hypertension of the newborn, congenital diaphragmatic hernia), both groups were represented in survivors and non-survivors. As both groups had received prolonged mechanical ventilation and had been exposed to high concentrations of oxygen before being placed on ECMO, pulmonary inflammation was also present in both groups. The data support the observation that a favourable outcome of pulmonary inflammation is associated with enhanced neutrophil apoptosis.

There is evidence from our previous in vitro studies that inhibition of neutrophil apoptosis by a range of cytokines and bacterial products $^{35}$ is associated with preservation of neutrophil proinflammatory functions-for example, respiratory burst, degranulation-and thus of the capacity to cause bystander tissue injury. ${ }^{35}$ An animal model of chronic inflammation (bleomycin injury) has also produced evidence for neutrophil activation up to 21 days after a pulmonary insult. $^{26}$

In the RDS group, the maximum apoptosis inducing activity of BAL fluid was detected on days 1 and 2, whereas the "wave" of apoptotic neutrophils was detected between days 4 and 10. The reasons for this time lag are unclear but may reflect the greatly extended life span of inflammatory compared with peripheral blood neutrophils. In animal models the half life of inflammatory neutrophils has been estimated to be several days, ${ }^{26}$ so that even where the rate of apoptosis is significantly accelerated there may be a lag between elaboration of a proapoptotic factor in BAL fluid and the detection of increased numbers of apoptotic cells.

The current data also support a major role for apoptosis in the clearance of neutrophils from the lung in neonatal pulmonary inflammation. For instance, the total neutrophil counts in the RDS group decreased from a mean of $176 \times 10^{4} /$ $\mathrm{ml}$ at day 7 to $40 \times 10^{4} / \mathrm{ml}$ on day 10 , concomitant with the identification of up to $17 \%$ apoptotic neutrophils in the BAL fluid.

Matute-Bello et al studied neutrophil apoptosis in ARDS and showed that neutrophil apoptosis was detected at low levels in BAL throughout the course of ARDS. ${ }^{7}$ Moreover, BAL fluid from ARDS patients suppressed constitutive neutrophil apoptosis, whereas fluid from normal volunteers did not. ${ }^{7}$ This inappropriate suppression of neutrophil apoptosis is 
analogous to the findings in our CLD group and was abrogated by blocking antibodies to G-CSF (granulocyte colony stimulating factor) and GM-CSF. ${ }^{7}$ In that study the investigators were unable to show an association between higher rates of neutrophil apoptosis and survival, although mean apoptosis was higher in the survivors than in those who died $(2.4 \% \vee 1.8 \%)$.

Increased numbers of macrophages were noted in the RDS group at day 4 (fig 2A), preceding the increase in apoptotic neutrophils that was evident on day 7 (fig $2 \mathrm{~B}$ ). It is conceivable that the alveolar macrophages play a role in induction of neutrophil apoptosis that is likely to be in addition to the proapoptotic effect of BAL on days 1 and 2 . Potential mechanisms for such induction by alveolar macrophages might include cell surface expression of death ligands-for example, Fas ${ }^{13}$ or TRAIL (tumour necrosis factor related apoptosis inducing ligand $)^{27}$ - which are proapoptotic for neutrophils and are known to be expressed on macrophage populations. ${ }^{28} 29$ In addition, these macrophages may play a role in clearance of apoptotic neutrophils. We attempted to quantify ingested apoptotic bodies and were able to detect examples (fig 3A), but at insufficient levels for a valid comparison of the RDS and CLD groups. A probable explanation is that light microscopic evaluation is substantially less sensitive than other methods-for example, TUNEL staining (which we found to be superior in unrelated studies on murine models of pulmonary inflammation) or electron microscopy. ${ }^{7}$ Alternatively, clearance of macrophages that have ingested apoptotic cells may occur rapidly through lymphatics. ${ }^{30}$ In the present studies, only two cytospins were available for each data point. In future studies, we hope to obtain additional cytospin preparations for immunohistochemistry (for macrophage death receptor expression) and TUNEL staining (for ingested apoptotic cells). Unfortunately, total cell numbers are too small for flow cytometry to be feasible for the majority of time points.

We considered whether the observed differences in BAL fluid modulation of apoptosis might be attributable to other factors to which the babies were exposed. Corticosteroids are known to inhibit neutrophil apoptosis, but at higher concentrations than are likely to be present in BAL fluid after antenatal dexamethasone treatment. ${ }^{31}$ None of the infants in the study received postnatal corticosteroids. Hypoxia has also been shown to prolong neutrophil survival, but at levels of hypoxia of $<1 \%$ oxygen. ${ }^{32}$ The degree of respiratory failure was similar between the two groups at the onset of ventilation, and tissue oxygenation was maintained in most cases, so this is unlikely to be relevant to our studies. The volume of fluid returned after the BAL procedure was similar between the CLD and RDS groups. In accordance to the recent recommendations of the European Respiratory Society's task force on bronchoalveolar lavage studies in children, we did not normalise for dilution of BAL fluid by using urea, secretory component of $\operatorname{IgA}$, or albumin. ${ }^{33}$ Although one potential factor that may modulate neutrophil apoptosis is surfactant, the use of exogenous surfactant was similar between the two groups (CLD 92\%, RDS 84\% (NS)). Furthermore, previous studies with peripheral blood neutrophils have not suggested a major proapoptotic effect of surfactant. ${ }^{34}$

One clear difference between the CLD and RDS groups was that the CLD group was significantly more premature (median gestation 26 weeks compared with 31 weeks). It is possible that, with increasing prematurity, neutrophils are more resistant to apoptosis. We noted a relation between the apoptosis inducing effect of the first available BAL fluid sample from each infant and gestation but not birth weight. It needs to be clarified further whether the data observed reflect immature neutrophil apoptotic mechanisms in pre- term infants, who are thus less likely to clear their neutrophils effectively. Allgaier et al have studied neutrophils purified from umbilical cord blood of neonates and have shown that they undergo apoptosis at a slower constitutive rate than adult neutrophils, and also that they are more resistant to the effects of the ligation of the Fas death receptor. ${ }^{35}$ It is possible, therefore, that age related differences in apoptosis susceptibility may influence the results observed. Nonetheless, we have shown clear differences in the effects of BAL fluid of CLD and RDS patients upon apoptosis of healthy adult neutrophils. This is most probably attributable to the presence or absence of soluble modulators of apoptosis. Prematurity could also be associated with inability to elaborate a proapoptotic factor or factors in the lung.

Within the limitations of the sample volumes obtained we attempted to identify soluble factors that might influence rates of apoptosis. In general, the ability of cytokines and other inflammatory mediators to modulate neutrophil apoptosis is well recognised, ${ }^{56}$ and many of these factors are present in BAL fluid in both adults ${ }^{76}$ and neonates with RDS. ${ }^{9-11}{ }^{37}$ Raised levels of GM-CSF have been associated with $\mathrm{ARDS}^{735}$ and CLD, ${ }^{22}$ and blocking antibodies have been shown to abrogate the antiapoptotic effect of BAL fluid from ARDS. ${ }^{7}$ Other studies, however, have not found this association, ${ }^{38}$ but have instead shown increases in IL-8 and G-CSF (the latter also found by Matute-Bello ${ }^{7}$ ). A cohort study suggested that levels of G-CSF or GM-CSF in BAL fluid were not, however, predictive of development of ARDS. ${ }^{36}$ We could not identify a correlation between GM-CSF levels and percentage neutrophil apoptosis, although a difference in GM-CSF levels between infants with RDS and CLD has previously been reported. ${ }^{22}$ The same was true for $\mathrm{TNF} \alpha$, which is known to be proapoptotic to human neutrophils at five hours, the time point used in our studies. ${ }^{18}$ Similarly, levels of IL-10-which are increased in ARDS $^{39} 40$ and are known to inhibit cytokine mediated delay of neutrophil apoptosis ${ }^{19}$ - were not associated with rates of apoptosis or with different patient groups. A recent report by Oei and colleagues did not find a relation between IL-10 in tracheal fluid and neutrophil apoptosis in preterm infants at risk of developing CLD. ${ }^{41}$ The lack of association of any single factor with the overall percentage of neutrophil apoptosis is not surprising, given the number of cytokines shown to be present in the inflamed lung. Identification of factors that correlate with outcome would, however, be of potential clinical use. Other potential factors that may modulate the neutrophil apoptotic process include Fas ligand ${ }^{42}$ and IL- $2{ }^{43}$ but we were unable to investigate these factors further because of the limited availability of BAL fluid from ventilated preterm infants.

\section{Conclusions}

These studies have shown that persistent airway neutrophilia in chronic lung disease is associated with inappropriately low rates of neutrophil apoptosis and failure to generate a proapoptotic BAL fluid. Conversely, resolution of lung inflammation in the RDS group is associated with higher rates of apoptosis and proapoptotic BAL fluid. These findings suggest that clinical outcome might be predictable from assessment of BAL fluid early in the development of ARDS and also that strategies to induce neutrophil apoptosis could be associated with improved outcome.

\section{ACKNOWLEDGEMENTS}

This study was supported by project grants from Trent NHS Research and Development to RAL, Action Research for AEC, and the British Lung Foundation for JRV. We would also like to thank the parents and staff on the neonatal unit at the Leicester Royal Infirmary and the Heartlink ECMO centre. 
Authors' affiliations

S Kotecha, J R Vyas, A E Currie, Department of Child Health, University of Leicester, Leicester, UK

R J Mildner, Heartlink ECMO Centre, Glenfield Hospital, Leicester L R Prince, R A Lawson, M K B Whyte, Respiratory Medicine Unit, Division of Genomic Medicine, University of Sheffield, Sheffield, UK

\section{REFERENCES}

1 Savill JS, Wyllie AH, Henson JE, et al. Phagocytosis of aged human neutrophils by macrophages is mediated by a novel "charge-sensitive" recognition mechanism. J Clin Invest 1989;84:1518-27.

2 Whyte M, Renshaw S, Lawson R, et al. Apoptosis and the regulation of neutrophil lifespan. Biochem Soc Trans 1999:27:802-7.

3 Whyte MK, Meagher LC, MacDermot J, et al. Impairment of function in aging neutrophils is associated with apoptosis. J Immunol 1993;150:5124-34.

4 Jones HA, Clark RJ, Rhodes CG, et al. In vivo measurement of neutrophil activity in experimental lung inflammation. Am J Respir Crit Care Med 1994; 149:1635-9.

5 Lee $A$, Whyte MK, Haslett C. Inhibition of apoptosis and prolongation of neutrophil functional longevity by inflammatory mediators. J Leukoc Biol 1993:54:283-8.

6 Colotta F, Re F, Polentarutti N, et al. Modulation of granulocyte survival and programmed cell death by cytokines and bacterial products. Blood 1992;80:2012-20.

7 Matute-Bello G, Liles WC, Radella F, et al. Neutrophil apoptosis in the acute respiratory distress syndrome. Am J Respir Crit Care Med 1997; 156:1969-77.

8 Park WY, Goodman RB, Steinberg KP, et al. Cytokine balance in the lungs of patients with acute respiratory distress syndrome. Am J Respir Crit Care Med 2001:164:1896-903

9 Kotecha S, Chan B, Azam N, et al. Increase in interleukin-8 and soluble intercellular adhesion molecule-1 in bronchoalveolar lavage of premature infants with chronic lung disease. Arch Dis Child 1995;72:F90-6.

10 Kotecha S, Wilson L, Wangoo A, et al. Increase in interleukin-1 beta and interleukin- 6 in bronchoalveolar lavage fluid obtained from infants with chronic lung disease of prematurity. Pediatr Res 1996;40:250-6.

11 Kotecha $S$, Wangoo A, Silverman $M$, et al. Increase in transforming growth factor- $\beta_{1}$ concentration in bronchoalveolar lavage fluid obtained from infants with chronic lung disease of prematurity. J Pediatr 1996;128:464-9.

12 Haslett C, Guthrie LA, Kopaniak MM, et al. Modulation of multiple neutrophil functions by preparative methods or trace concentrations of bacterial lipopolysaccharide. Am J Pathol 1985;1 19:101-10.

13 Renshaw SA, Timmons SJ, Eaton V, et al. Inflammatory neutrophils retain susceptibility to apoptosis mediated via the Fas death receptor. J Leukoc Biol 2000;67:662-8.

14 Usher LR, Lawson RA, Geary I, et al. Induction of neutrophil apoptosis by the Pseudomonas aeruginosa exotoxin pyocyanin: a potential mechanism of persistent infection. J Immunol 2002;168:1861-8.

15 Homburg $\mathrm{CH}$, de Haas $\mathrm{M}$, von dem Borne $\mathrm{AE}$, et al. Human neutrophils lose their surface Fc gamma Rlll and acquire Annexin $\mathrm{V}$ binding sites during apoptosis in vitro. Blood 1995;85:532-40.

16 Dransfield I, Buckle AM, Savill JS, et al. Neutrophil apoptosis is associated with a reduction in CD16 (Fc gamma RIII) expression. J Immunol 1994; 153:1254-63.

17 Mildner RJ, Killer HM, Firmin RK, et al. Resolution of acute lung inflammation in infants treated receiving ECMO [abstract]. Am J Respir Crit Care Med 1999:159:A153.

18 Murray J, Barbara JA, Dunkley SA, et al. Regulation of neutrophil apoptosis by tumor necrosis factor-alpha: requirement for TNFR55 and TNFR75 for induction of apoptosis in vitro. Blood 1997;90:2772-83.

19 Keel M, Ungethum U, Steckholzer U, et al. Interleukin-10 counter regulates proinflammatory cytokine-induced inhibition of neutrophil apoptosis during severe sepsis. Blood 1997; 90:3356-63.

20 Cox G. IL-10 enhances resolution of pulmonary inflammation in vivo by promoting apoptosis of neutrophils. Am J Physiol 1996;271:L566-71.
21 Glynn P, Coakley R, Kilgallen I, et al. Circulating interleukin 6 and interleukin 10 in community acquired pneumonia. Thorax 1999;54:51-5.

22 Bry K, Hallman M, Teramo K, et al. Granulocyte-macrophage colonystimulating factor in amniotic fluid and in airway specimens of newborn infants. Pediatr Res 1997:41:105-9.

23 Murch SH, MacDonald TT, Wood CB, et al. Tumour necrosis factor in the bronchoalveolar secretions of infants with the respiratory distress syndrome and the effect of dexamethasone treatment. Thorax 1992;47:44-7.

24 Blahnik MJ, Ramanathan R, Riley CR, et al. Lipopolysaccharide-induced tumor necrosis factor-alpha and IL-10 production by lung macrophages from preterm and term neonates. Pediatr Res 2001;50:726-31.

25 Ogden BE, Murphy SA, Saunders GC, et al. Neonatal lung neutrophils and elastase/proteinase inhibitor imbalance. Am Rev Respir Dis 1984; 130:817-21.

26 Jones HA, Clark RJ, Rhodes CG, et al. In vivo measurement of neutrophil activity in experimental lung inflammation. Am J Respir Crit Care Med 1994; 149:1635-9.

27 Renshaw SA, Parmar JS, Singleton V, et al. Acceleration of human neutrophil apoptosis by tumor necrosis factor-related apoptosis-inducing ligand (TRAIL). J Immunol 2003;170:1027-33.

28 Brown SB, Savill J. Phagocytosis triggers macrophage release of Fas ligand and induces apoptosis of bystander leukocytes. J Immunol 1999;162:480-5.

29 Halaas O, Vik R, Ashkenazi A, et al. Lipopolysaccharide induces expression of APO2 ligand/TRAIL in human monocytes and macrophages. Scand J Immunol 2000;51:244-50.

30 Bellingan GJ, Caldwell $\mathrm{H}$, Howie SE, et al. In vivo fate of the inflammatory macrophage during the resolution of inflammation: inflammatory macrophages do not die locally, but emigrate to the draining lymph nodes. J Immunol 1996;157:2577-85.

31 Meagher LC, Cousin JM, Seckl JR, et al. Opposing effects of glucocorticoids on the rate of apoptosis in neutrophilic and eosinophilic granulocytes. J Immunol 1996;156:4422-8.

32 Hannah S, Mecklenburgh K, Rahman I, et al. Hypoxia prolongs neutrophil survival in vitro. FEBS Lett 1995;372:233-7.

33 de Blic J, Midulla F, Barbato A, et al. Bronchoalveolar lavage in children. ERS Task Force on bronchoalveolar lavage in children. European Respiratory Society. Eur Respir J 2000;15:217-31.

34 Suwabe A, Otake K, Yakuwa N, et al. Artificial surfactant (Surfactant TA) modulates adherence and super-oxide production of neutrophils. Am J Respir Crit Care Med 1998;158:1890-9.

35 Allgaier B, Shi M, Luo D, et al. Spontaneous and Fas-mediated apoptosis are diminished in umbilical cord blood neutrophils compared with adult neutrophils. J Leukoc Biol 1998;64:331-6.

36 Matute-Bello G, Liles WC, Radella F, et al. Modulation of neutrophil apoptosis by granulocyte colony-stimulating factor and granulocyte/macrophage colony-stimulating factor during the course of acute respiratory distress syndrome. Crit Care Med 2000;28:1-7.

37 Currie AE, Vyas J, MacDonald J, et al. Epidermal growth factor in the lungs of infants developing chronic lung disease of prematurity. Eur Respir J $2001 ; 16: 796-800$

38 Aggarwal A, Baker CS, Evans TW, et al. G-CSF and IL-8 but not GM-CSF correlate with severity of pulmonary neutrophilia in acute respiratory distress syndrome. Eur Respir J 2000;15:895-901.

39 Armstrong L, Millar AB. Relative production of tumour necrosis factor alpha and interleukin 10 in adult respiratory distress syndrome. Thorax 1997:52:442-6.

40 Parsons PE, Moss M, et al. Circulating IL-1 ra and IL-10 levels are increased but do not predict the development of acute respiratory distress syndrome in at-risk patients. Am J Respir Crit Care Med 1997;155:1469-73.

41 Oei J, Wang H, Henry R. decreased neutrophil apoptosis in tracheal fluids of preterm infants at risk of chronic lung disease. Arch Dis Child 2003;88:F245-9.

42 Serrao KL, Fortenberry JD, Owens ML, et al. Neutrophils induce apoptosis of lung epithelial cells via release of soluble Fas ligand. Am J Physiol 2001;280:L298-305.

43 Lesur O, Kokis A, Hermans C, et al. Interleukin-2 involvement in early acute respiratory distress syndrome: relationship with polymorphonuclear neutrophil apoptosis and patient survival. Crit Care Med 2000;28:3814-22. 